\title{
Tactics to Optimize the Potential and CardioBioFeedback in Stress Management: The French Experience
}

\author{
Marion Trousselard, MD, PhD, ${ }^{1, *}$ Frédéric Dutheil, $\mathrm{MD}, \mathrm{PhD}{ }^{2}$ Marie-Hélène Ferrer, $\mathrm{MD}, \mathrm{PhD}$, ${ }^{1, *}$ \\ Nathalie Babouraj, $\mathrm{MD}^{3}$ and Frédéric Canini, $\mathrm{MD}, \mathrm{PhD}^{1, *}$
}

\begin{abstract}
Background: Chronic work-related stress is a well-known public health problem, and it concerns Military populations. Stress responses lead to specific changes in immunity, physiologic systems, and regulation of stress hormones. Overexposure to stress has an important role in the development and courses of mental diseases, as well as being a factor in increased abdominal obesity, osteoporosis, infections, and cardiovascular problems. The aim of this research was to evaluate the effectiveness of two stress psychologic fitness management programs based on a mind-body approach to stress perception and stress reactivity in a Military population subjected to daily operational stress.

Method: 180 young military fire fighter recruits were randomly assigned to a controlled intervention trial including three groups: a cognitive stress program (tactics to Optimized the Potential - TOP) group, an emotional CardioBioFeedback stress program (CBF) group, or a control group. The stress programs training lasted eight weeks, with one hour training per week. A placebo was administered as a nutriceutical in all three groups during the time of the training. Primary outcome variables included the perceived stress; second outcome variables included stress reactivity (mindfulness, negative mood, Immunoglobulin A levels). Post intervention effectiveness on the long-term programs' benefits was evaluated.

Results: Both TOP and CBF stress programs reduce operational stress in military population. Although the size of the effects was small, they must be considered at the clinical level. Long-term effects depend on the frequency of daily practice. Results were discussed with reference to mind-body theory.

Conclusions: Short stress programs intervention improved stress perception and stress reactivity in healthy workers. Recommendations about mind-body interventions were proposed for the military population.
\end{abstract}

Key Words: Mind-Body Intervention Training, Anxiety, Mindfulness, Perceived Stress

\footnotetext{
${ }^{1}$ Senior Researcher, Institute of Biomedical Research, Armies' Health Service, Bretigny sur Orge, France.

${ }^{2}$ Researcher, Laboratory of Metabolic Adaptations to Exercise in Physiological and Pathological Conditions, EA3533, Blaise Pascal University, Clermont-Ferrand, France.

${ }^{3}$ Physician, Paris Fire Brigade, France.

This article was prepared as part of the work product of NATO Science and Technology Organization, Task Force, Human Factors and Medicine (STO-TR-HFM) 195, Integrative Medicine Interventions for Military Personnel.

*The opinions and assertions contained herein are the private views of the authors and are not to be construed as official or as reflecting the views of the French Armed Forces Biomedical Research Institute or the French Military Health Service at large. The authors indicate that they do not have any conflicts of interests.
} 


\section{INTRODUCTION}

C HRONIC STRESS AT WORK is a well-known public health problem, which also concerns Military populations. ${ }^{1-3}$ Stress responses lead to specific changes in immunity, ${ }^{4-6}$ physiologic systems, ${ }^{7,8}$ and regulation of stress hormones. ${ }^{9,10}$ Overexposure to stress has an important role in the development and courses of mental diseases, as well as being a factor in increased abdominal obesity, osteoporosis, infections, and cardiovascular problems. ${ }^{11}$

Among psychologic fitness treatment protocols, heart coherence, a CardioBioFeedback (CBF) approach, relies on the fact that heart rhythm becomes more erratic with negative emotions, such as anger or frustration stability, ${ }^{12}$ and becomes more ordered/coherent with sustained positive emotions, such as appreciation, love, or compassion, ${ }^{12}$ leading to optimal performance and well-being. ${ }^{13,14}$ The CBF program uses feedback from a simple pulse sensor to reflect changes in emotional/psychologic states ${ }^{8,15}$ and to enable users to learn to reduce stress and stabilize their emotions. CBF is easy to learn and use; it is also cost-effective.

Another treatment protocol for psychologic fitness, developed by the French Army, focuses on cognitive training to regulate emotions, using Tactics to Optimize the Potential (TOP). TOP involves learning easy techniques of mental skill to improve cognitively based problem-solving ${ }^{16,17}$ through control of respiration, by relaxation, ${ }^{18-22}$ and by using visualization. ${ }^{23,24}$ These task-oriented coping strategies improve performance ${ }^{25,26}$ and health. ${ }^{27}$ TOP is widely practiced in air-traffic controllers, with apparently good results.*

Among Military populations, Military firefighters (FFs) in Paris, France (PFFs) appear to be particularly exposed to stress and could benefit from TOP intervention. ${ }^{28}$ To test the current authors' concept that stress-management programs (SMPs) would benefit this group, it was hypothesized that: (1) young recruits aiming to become PFFs would be particularly exposed to stress; (2) SMPs would be effective for reducing perceived stress ${ }^{29}$; and (3) the effects of SMPs would result in lowered immunoglobulin(Ig)A levels.

The first aim of the study was to evaluate the effectiveness of SMPs on perceived stress (the primary outcome variable). Perceived stress is sensitivity to chronic stress stemming from a lifetime of negative circumstances, stress concerning expectations with regard to future events, and have stress reactions to specific events, which included events experienced by PFFs. ${ }^{30}$

The second aim of the study was to evaluate SMPs' effectiveness on negative mood, mindfulness, and immunity (secondary outcome variables). A comparison between the two SMPs (TOP and CBF) was assessed. Finally, a longterm follow-up was performed to investigate the influence of frequency of daily practice on SMP effectiveness.

\footnotetext{
*Information from French report from French Joint staff chief
} No. 803/DEF/EMA/RH/FORM/2013.

\section{MATERIALS AND METHODS}

\section{Participants}

New professional volunteer fire fighters (FFs) were recruited from the formation center of the French Army's PFFs unit. The ethics committees from the Health Army of Paris approved the study. To be eligible, participants were males with: no endocrine disease; no recent extraprofessional life-stress events (such as death of a near relative, divorce); no current illness; no use of medications to modulate inflammatory diseases (corticosteroids, antiinflammatory drugs, immunomodulatory drugs); no use of medications with chronotropic effects taken over the previous 6 months (B-blockers, diltiazem, verapamil, anxiolytics, or antidepressants).

\section{Measurements}

Primary outcome: Using the Perceived Stress Scale (PSS; 29,30 ), this variable was measured with 14 items rated on a 5 -point scale that ranges from (1) "never" to (5) "often." Higher scores indicate higher perceived stress. Two subfactors were calculated: (1) vulnerability or "perceived distress" and (2) uncontrolled perceived stress. ${ }^{30}$ With respect to anxiety and depression predictivity, stress thresholds have been considered to be pertinent above $27 .{ }^{31}$

Secondary outcomes: Mood was evaluated using an abbreviated version of the Profile of Mood States (POMS). ${ }^{32}$ It consisted in an adjective checklist of 37 items rated on a 5point scale that ranged from (1) "not at all" to (5) "extremely." Each subject was asked to answer according his present mood. Six factors were then calculated: (1) anxietytension; (2) depression-dejection; (3) anger-hostility; (4) fatigue-inertia; (5) vigor-activity; and (6) confusionbewilderment. For a negative mood variable, an index was calculated for each subject separately by adding the scores in the mood subscales without activity/vigor.

Mindfulness levels were assessed using the French version of the short form of the Freiburg Mindfulness Inventory-14 (FMI-14), which is a 14-item, 4-point (1-4) self-report questionnaire developed for people with no background knowledge about mindfulness. ${ }^{33,34}$ The FMI-14 is consistent and reliable scale for evaluating several important aspects of mindfulness and is considered to be onedimensional for practical purposes. ${ }^{34}$ Depending on the suggested timeframe in the instructions, this inventory can be used to assess state- or trait-like components. ${ }^{34}$ For the purpose of this study, this short form was used for measuring respondents' state of mindfulness. Higher scores indicated higher levels of mindfulness.

Immunity assessments concerned salivary IgAs, which are antibodies that collectively play a critical role in mucosal immunity. They are used as immunologic stress markers. ${ }^{35,36}$ For each immunity measure, a 5 -mL saliva sample was collected in Salivette tubes according to 
specifications of the provider (Salimetrics, Europe Ltd.). Two hours before each collection, no eating, drinking, or smoking was allowed. Once filled, the tubes were centrifuged, sampled into $1.5-\mathrm{mL}$ aliquots stored at $-80^{\circ} \mathrm{C}$ until analysis. Salivary concentrations were analyzed using enzyme-linked immunosorbent assay kits (Kit Salimetrics LL-C, PA). All samples were analyzed in duplicate.

\section{Compliance with the SMPs}

The PFFs' compliance with the protocol was assessed by the frequency of daily practice, which was evaluated with a visual analogue scale (VAS) at month (M)12 and M18. Subjects had to respond to the following question: "Can you indicate the frequency of your daily practice on the bottom line?" The VAS was a horizontal, noncalibrated line of $100 \mathrm{~mm}$, ranging from very low (0; no daily practice) to very high (100; more than three daily exercises of 3 minutes each) daily practice.

\section{Protocol}

The French Army's PFFs unit includes new PFFs each month for a selective instruction time period of 6 months. New PFFs have a 1-year contract beginning with a 6-month formation (M0-M6) followed by a 6-month active-duty period (M6-M12). The 6-month formation starts with a 2month Military formation (M1 and M2), followed by a 2month training in providing emergency relief for victims (M3 and M4), and then by a 2-month specific FF formation (M5 and M6). Within the current study, volunteers were included at M4 (baseline measurements) after the first 4-month formation, because most of the young recruits fail during this period and are withdrawn from the contract. Included participants were randomly assigned (computer-generated randomization) without stratification to one of the three following groups: TOP, CBF, control. A similar number of PFFs were enrolled into each group at each time. Psychologic training (TOP and $\mathrm{CBF}$ ) was performed during the last 2month (months 5 and 6 ) period of specific FFs' formation).

The three groups received a placebo as a nutraceutical to mitigate the placebo effect. The placebo consisted of 1 starch capsule per day during the last 2-month period of formation (when the SMP occurred; Fig. 1). Approval for placebo use was received from the French agency for evaluation of medicinal products and safety security. Measuring the number of capsules taken during the program session at months 5 and 6 assessed compliance with the placebo.

Participants were included at M4 within those succeeding the 2-month Military formation and the 2-month training in providing emergency relief for victims. All baseline assessments (M4) were repeated at the end of the SMPs (M6), at the end of the 6-month active duty period (M12), and at M18, with the exception of IgAs, which were only assessed at M4 and M6 (Fig. 1). All assessments were conducted between 15:30 hours (Military time) and at 18:00 hours to avoid circadian variations. ${ }^{37}$

\section{Statistical Analyses}

Enrollment was set to end when 180 patients (60 per group) were included in the study. It was estimated that, with 55 participants in each group, the study would have $>80 \%$ power to detect a clinically important difference among the groups in change of perceived stress, assuming a mean between-group difference of 2 points from the control group, with a pooled standard deviation (SD) of 2.1 (on the basis of preliminary data), at an $\alpha$ level of $5 \%$.

For the POMS, a negative mood index was calculated for each PFF separately by adding the score in the mood subscales anxiety-tension, depression-dejection, anger-hostility, fatigue-inertia, and confusion-bewilderment without the activity/vigor subscale.

Baseline characteristics were compared among the groups using an analysis of variance (ANOVA). Intentionto-treat (ITT) analyses were performed to assess the effectiveness of the SMPs. Effects of the groups' programs were evaluated using ANOVAs on the changes in outcome variables. Change scores were calculated as change by subtracting baseline (M4) scores from scores at the end of the programs (M6). This was done for each outcome variable separately. Comparisons between $\mathrm{CBF}$ and TOP programs

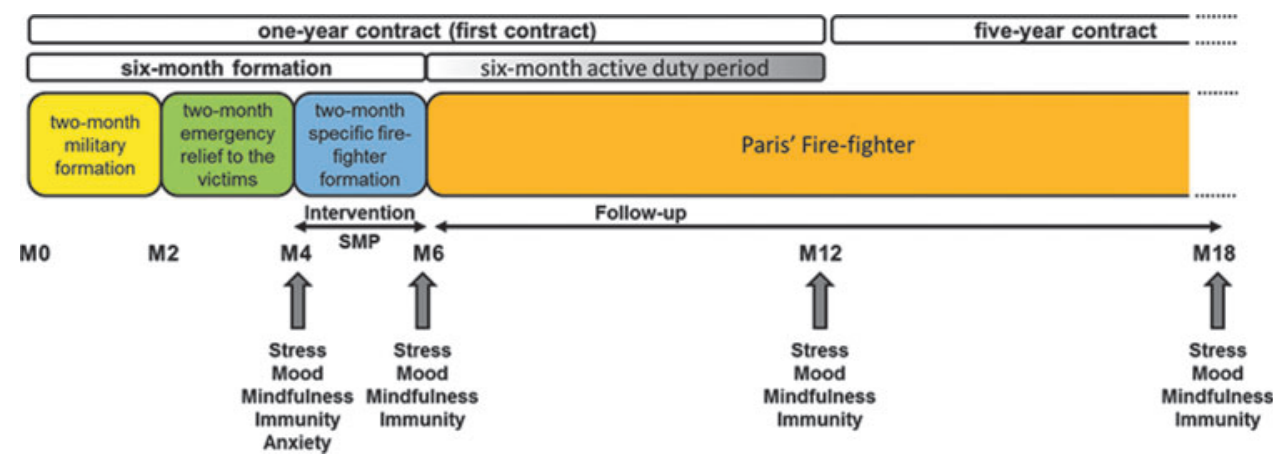

FIG. 1. Study design. SMP, stress management program. M, month. 
were evaluated by posthoc analyses, when results were significant among the groups. Cohen's $d$ was used for estimating sample sizes.

For long-term effects (M12 and M18), CBF and TOP groups were pooled in an SMP group for comparisons to controls. The analyses were performed per treatment protocol. Repeated measures ANOVAs were applied on each outcome variable score separately. Regression equations were performed to determine the impact of the daily practice scores.

Data are presented in this article as mean percentage change $\pm S D$, unless otherwise specified. Analyses were performed with SPSS, v17.0. The current authors judged $P<0.05$ as being significant and $P \leq 0.1$ as a tendency toward a difference.

\section{RESULTS}

\section{Participants}

For this study, 180 PFFs were randomized into 3 groups of 60 PFFs each. Fourteen PFFs (7.78\%) were excluded because they had traumatic injuries between inclusion in the study and the beginning of the SMPs (Fig. 2). No dropouts were recorded between M4 and M6. Eighty-four completed the entire 14-month study. A comparison between participants withdrawing from the study and those who remained showed no difference in descriptive characteristics, independent of the time at which they withdrew.

The percentage of compliance was $\sim 75 \%$ with a mean number of taken capsules of 44.32 between M4 and M6 (73.86\%) for the TOP group, 45.58 (75.96\%) for the CBF, and 44.5 (74.16\%) for the control group. Compliance did not differ among the groups.

Briefly, participants were males, ages 18-25, mainly Caucasian, with $78.3 \%$ not engaged and half with university diplomas. All PFFs practiced at least 4 hours per week of sports and $16.1 \%$ were smokers. All PFFs practiced sports with a mean number of sports training per day of 6 hours. No difference was observed between groups at baseline (Table 1). Eighty seven percent of PFFs were considered to be stressed at baseline and were above the clinical threshold of $27 .{ }^{31}$

\section{Main Outcome}

The score of perceived stress change differed among the groups $(F=2.7 ; P=0.05)$. The TOP group had a higher decrease than the controls $(P=0.05)$ and tended to differ from the CBF group ( $P=0.09 ;$ Fig. 3$)$. No differences were observed in PSS subfactors change. However, at M6, subjects in the TOP program tended to have smaller scores on the uncontrolled subfactor than subjects in the CBF program $(F=2.88 ; P=0.09)$.

The strength of the phenomenon was small, with $d=0.18$ for TOP and $d=0.15$ for CBF. The number of PFFs decreasing their scores under the clinical threshold was higher in the SMP groups (7.8\%) than in the control group $(0 ; 7$ PFFs from the TOP group; and 7 PFFs from the CBF group versus 0 PFFs in the control group, $\chi^{2}=4.45 ; P=0.03$; i.e. a $12 \%$-reduction of perceived stress occurred in the SMP groups, compared with the control group). The number needed to treat (NNT) with the intervention (CBF and TOP) to see a one-person benefit (under the threshold) is 9 .

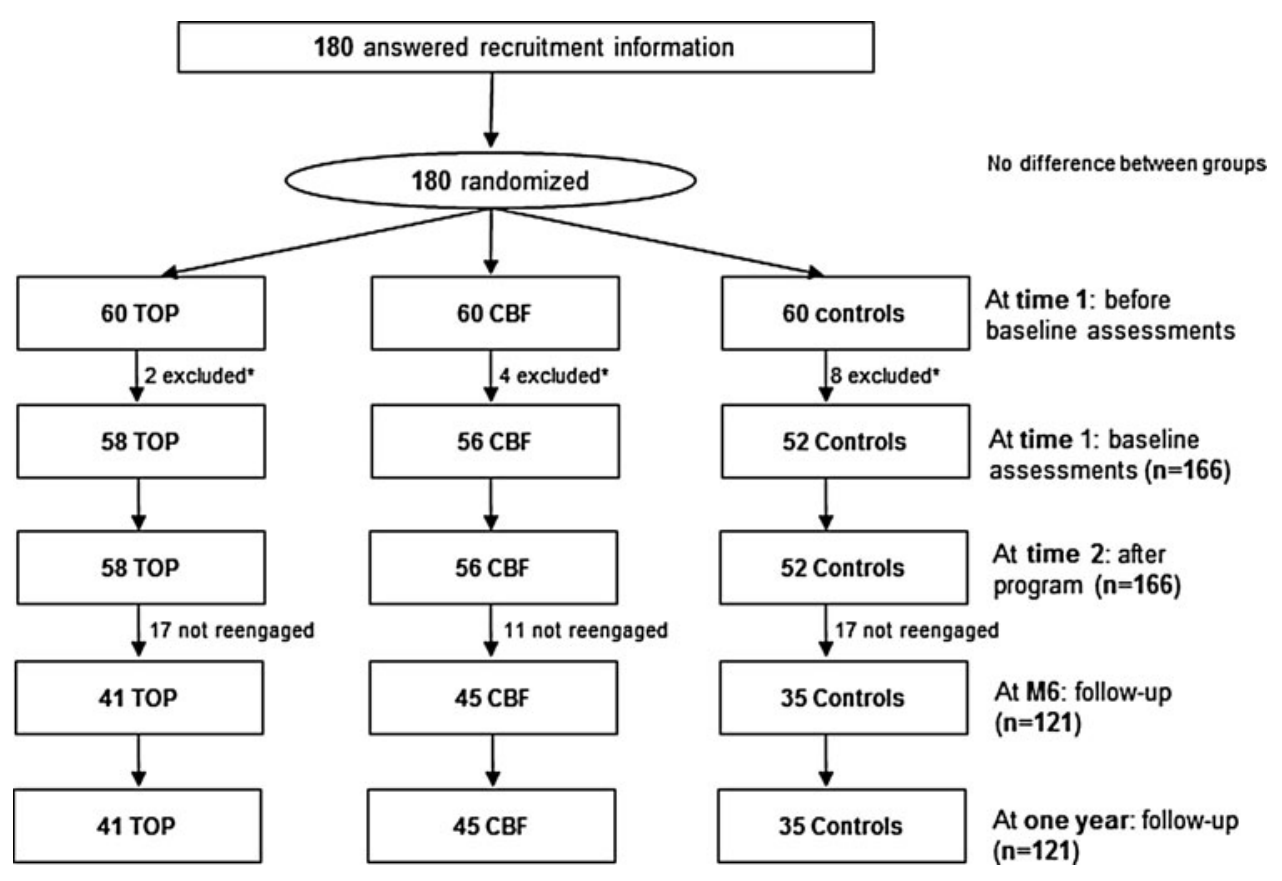

FIG. 2. Flowchart. TOP, Tactics to Optimize the Potential; CBF, CardioBioFeedback; M, month. 
Table 1. Means and SDs at Baseline (M2) and at M4 for Full Group and each Randomized Group

\begin{tabular}{|c|c|c|c|c|c|}
\hline Outcomes & Full group & Gr TOP & Gr $C B F$ & Gr Control & $F(\mathrm{P})$ \\
\hline \multicolumn{6}{|l|}{ Primary outcome } \\
\hline M2 Perceived stress & $34.81(7.4)$ & $34.86(6.9)$ & $35.03(7.1)$ & $33.41(8.5)$ & $0.77(0.46)$ \\
\hline M4 perceived stress & $33.27(6.79)$ & $32.43(6.39)$ & $33.48(7.31)$ & $33.39(6.71)$ & $0.70(0.49)$ \\
\hline M2 Uncontrolled stress & $17.7(4.1)$ & $17.71(3.7)$ & $17.93(3.6)$ & $17.55(4.7)$ & $0.28(0.75)$ \\
\hline M4 Uncontrolled stress & $16.76(3.69)$ & $16.25(3.23)$ & $17.14(3.78)$ & $16.91(4.07)$ & $1.32(0.2)$ \\
\hline M2 Distress & $17.11(3.9)$ & $17.35(3.6)$ & $17.11(3.8)$ & $16.86(4.3)$ & $0.52(0.59)$ \\
\hline M4 Distress & $16.29(4.01)$ & $16.19(4.34)$ & $16.3(4.32)$ & $16.2(3.38)$ & $0.13(0.87)$ \\
\hline \multicolumn{6}{|l|}{ Secondary outcomes } \\
\hline M2 Mindfulness & $40.4(5.4)$ & $41(5.4)$ & $39.1(5.4)$ & $41.1(5.2)$ & $2.53(0.09)$ \\
\hline M4 Mindfulness & $40.28(4.89)$ & $40.85(4.7)$ & $39.23(5.47)$ & $40.78(4.5)$ & $1.09(0.34)$ \\
\hline M2 Negative mood index & $18.1(17.1)$ & $17.3(14.5)$ & $19.7(20.4)$ & $17.4(16)$ & $0.29(0.74)$ \\
\hline M4 Negative mood index & $15.95(16.51)$ & $16.63(15.23)$ & $18.98(15.07)$ & $15.21(16.28)$ & $0.65(0.49)$ \\
\hline $\mathrm{M} 2 \operatorname{IgA}(\mu \mathrm{g} / \mathrm{mL})$ & $149.1(91.6)$ & $132.4(88.2)$ & $152.1(87.08)$ & $163.6(99.7)$ & $0.37(0.68)$ \\
\hline M4 IgA $(\mu \mathrm{g} / \mathrm{mL})$ & $118.24(38.36)$ & $105.17(35.56)$ & $135.59(32.02)$ & $113.97(47.52)$ & $0.38(0.68)$ \\
\hline
\end{tabular}

SD, standard deviation; M, month; Gr, group; TOP, Tactics to Optimize the Potential; CBF, CardioBioFeedback; F, IgA, immunoglobulin A.

\section{Secondary Outcomes}

Changes in negative mood index differed among the groups $(F=3.32 ; P=0.04)$. The decrease was higher in the TOP group than in the control group $(p=0.04)$, higher in the $\mathrm{CBF}$ group than in the control group $(P=0.05)$, and tended to be higher in the TOP group than in the CBF group $(P=0.06$; Fig, 3$)$. The strength of the phenomenon was small-to-middle with $d=0.4$ for TOP and $d=0.1$ for CBF.

No differences were observed in mindfulness changes.

The IgA changes tended to differ between groups $(F=2.29 ; P=0.09)$ with a tendency to a smaller decrease for TOP and CBF programs than for the control group. Comparison between programs showed a tendency to a smaller decrease for the CBF program compared to the TOP program $(P=0.1$; Fig. 3$)$.

\section{Long-Term Effects (Table 2)}

A time effect was observed for perceived stress $(F=29.92 ; P=0.001$ ), with a higher score at M12 (the incertitude session), compared with other measurement timepoints. All PFFs were considered to be stressed at M12 and were above the clinical threshold of 27 , whereas $\sim 78 \%$ of the PFFs were above the clinical threshold at M18 (77.41 for the SMP group and 78.3 for the control group). No group effect was observed.

A time effect was also observed for mindfulness scores $(F=5.31, P=0.001)$, with scores higher at M12. Significant interaction among the groups $(F=3.32 ; P=0.02)$ revealed higher mindfulness scores in the SMP group than in the control group at M12.

Negative mood index had a time-effect tendency ( $F=2.27, P=0.08$ ), with higher scores at M18 (operational session). Interaction among the groups tended toward a lower negative mood index in the SMP groups than in the control group $(F=2.06 ; P=0.1$; Fig. 3$)$.

TOP and CBF groups did not differ in daily practice scores. If only $10 \%$ of the 62 PFFs from the SMP group did not fill out the VAS at M12 or at M18, the average of total daily practice in the SMP group would be 2.7/10 at M12 and 2.9/10 at M18. PFFs did not perform homework assignments without instructors, regardless of which SMP these PFFs were in. At M12, daily practice scores accounted for $17.9 \%$ of the variance in negative mood improvement $(F=2.974 ; P=0.03)$.
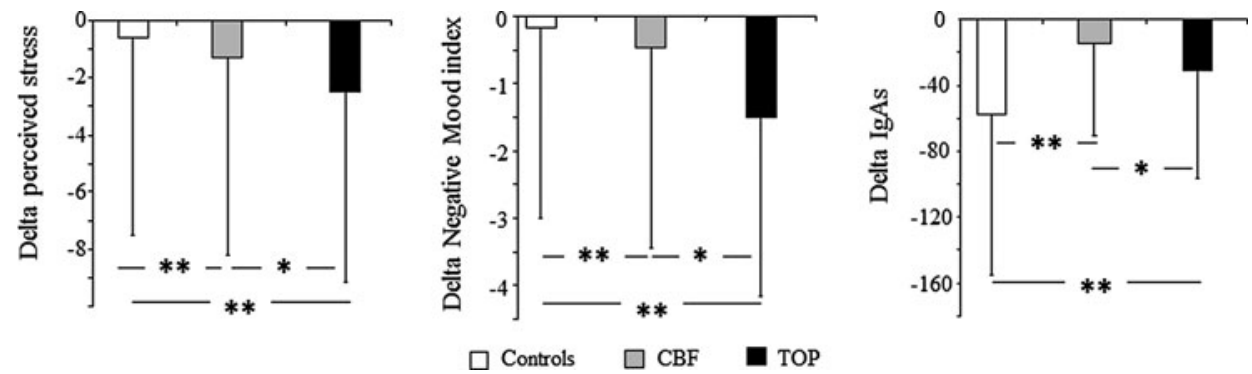

FIG. 3. Change in outcomes after the stress management program. CBF, CardioBioFeedback; TOP, Tactics to Optimize the Potential; IgAs, immunoglobulin As. ${ }^{*} p<0.05 ; * p<0.1$. 
Table 2. Means and SDs at M12 and M18 (Follow-UP) FOR The SMP AND CONTROL Groups

\begin{tabular}{lll}
\hline Instrument & SMP group & Control group \\
\hline Perceived Stress Scale & & \\
M12 $(\mathrm{N}=121)$ & $41.13(6.31)$ & $40.33(6.65)$ \\
M18 $(\mathrm{N}=121)$ & $32.98(7.6)$ & $32.47(9.7)$ \\
Negative mood index & & \\
M12 $(\mathrm{N}=121)$ & $17.15(15.97)$ & $23.26(21.95)$ \\
M18 $(\mathrm{N}=121)$ & $17.14(20.98)$ & $24.47(26.37)$ \\
Mindfulness & & \\
M12 $(\mathrm{N}=121)$ & $48.95(8.21)$ & $45.97(5.41)$ \\
M18 $(\mathrm{N}=121)$ & $41.31(5.24)$ & $41.65(6.95)$ \\
\hline
\end{tabular}

SD, standard deviation; M, month; SMP, stress management program.

\section{DISCUSSION}

The young Military PFFs in this study had high perceivedstress levels ${ }^{32,38,39}$ and were generally mindful. ${ }^{33,40}$ The results of this preliminary study showed a benefit for SMPs for treating stress perception, compared to the control condition in terms of NNT. The significant decrease in percent of subjects who fell under the clinical threshold after the SMPs was associated with a significant decrease in negative mood and better mucosal immunity status. This combination of statistically significant improvements in these diverse domains lends support to the clinical relevance of the improvements produced by the evaluated SMPs.

First, these results are important insofar as research has indicated that perceived stress is often linked to somatic distress; negative affect ${ }^{31,39}$; and psychologic disorders, such as anxiety and depression. ${ }^{32}$ Second, the decrease in negative mood was of interest, because it is well-known that negative mood is associated with physical and mental disorders ${ }^{41}$ mental diseases, ${ }^{41,42}$ and immune-mediated diseases. ${ }^{43}$ Third, the tendency of decreased IgAs after the PFF program sessions is in accordance with the difficulties of the Military formation reported by the PFFs for becoming an FF. A high level of perceived stress already has been associated with a low level of $\operatorname{IgA}$, namely in health care professionals. ${ }^{44}$

Organizations should be more concerned about workrelated stress, which may contribute to an increase in the incidence of infectious diseases and subsequent increases in days off work. Interestingly, perceived stress and negative mood index changes tend to be higher for the cognitivebased experiential training, whereas IgA decrease tended to be lower for the emotionally based experiential training, compared to the cognitive one or the absence of training. This result cannot be explained by a difference in sports, as the PFFs had similar sports practices during the initial PFF formation. The positive effect of CBF on IgA already has been demonstrated. ${ }^{45}$ The observed higher effect of the TOP on uncontrolled stress as on negative mood may be under- stood as an increase in the ability to evaluate a situation best. ${ }^{46,47}$ Whether or not differences between TOP and CBF warrant further investigations, an association between TOP and CBF may be found.

Long-term effects evaluation was based on an ecologic protocol characterized by an incertitude session followed by an operational one. The incertitude session appeared to be a real stress period, as indicated by the increase in perceived stress in the 3 groups. The intervention program did not appear to help subjects deal with uncertainty, as no difference was observed among the groups. Nevertheless, these subjects did have improved mindfulness. The concept of mindfulness is characterized by awareness and acceptance of experiences; flexible regulation of attention; objective receptivity to body experiences; and orientation to the hereand-now. ${ }^{34,48}$

This was of interest as it is well-known that high levels of mindfulness are believed to be associated with well-being, ${ }^{48}$ higher body satisfaction (Body Cathexis Scale) ${ }^{49}$; and better identification and description of feelings; while low mindfulness is associated with anxiety, depression, and difficulties in coping with stress. ${ }^{50,51}$ It can be proposed that the SMPs did not improve stress perception when the PFFs faced stressful situations but did help the PFFs to cope better with stressful situations by increasing mindfulness when necessary. In addition, it can be proposed that this mindfulness also produced some possible benefits on negative mood reactivity in more quiet situations, as was observed in during the operational session.

Altogether, these results must be considered in the mindbody connection framework, ${ }^{52-55}$ a function that highlights how the whole body is involved in emotions and feelings rather than just the brain. ${ }^{56}$ An SMP would be considered as a tool for increasing mind-body connection, which appears to be particularly efficient when environmental challenges require a person to cope with stress.

Furthermore, when considering these study results, one must take into account that the subjects did not practice regularly; one reason could be the decision of the newly promoted Military FF command during the study to introduce ethic formation in place of the SMPs. It is not clear if the subjects would have become more adept if they had Military support for the practice. Nevertheless, it is reasonable to conclude that greater benefits may accrue to participants if they engaged in daily practice.

\section{Strengths}

This study has some major strengths. It had a randomized, run-in design; compliance to SMPs was measured; there was a sufficient number of participants to detect differences among the groups; and the study had community-based long-term interventions. In addition, this study was unique in that both SMPs and PFFs have seldom been investigated 
in randomized controlled trials, using a combination of subjective and objective measures.

This study highlights, on the one hand, the effects of SMPs in an ecologic realistic environment, and, on the other hand, differences in benefits between cognitive- and emotionally based training.

\section{Limitations}

There were several limitations to this study. From a methodological perspective, it is not certain that giving the 3 groups a nutraceutical truly mitigated the placebo effect. From a clinical perspective, the clinical benefits were not evaluated with respect to the small size of the effects. The results cannot necessarily be generalized to all workers with occupational stress. Women were not included; however, using only a male population reduced the variability of measures. Dropouts were high, but many PFFs did not have their contracts renewed, and there was no headquarters' support during the follow-up period. Compliance may have appeared to be poor and may have decreased the benefits of the SMPs. Finally, cost-benefit in terms of incidence of infectious diseases, and increase of days off work as well as improvement in Military skills and operational performances were not evaluated.

\section{CONCLUSIONS}

Treatment of stress and its effects involves the whole person; body and brain are interconnected in both TOP and CBF SMPs and produced relief of operational stress in PFFs. To improve personnel performance and well-being, Military and civilian organizations should be more concerned about occupational stress through promotion of SMPs. Given that the body is involved in the working of the brain and the emotions, it is logical that treatment of diseases would also involve the whole person, given that the body and brain are interconnected.

\section{RECOMMENDATIONS}

Future studies of other stress-reducing interventions, such as meditation, Qigong, and yoga, appear to be pertinent for evaluating Military individuals. Furthermore, conducting interventional trials on stress-related diseases warrants further investigations. In accordance with such results, the main recommendation would be to develop professionals (e.g., trainers, educators) to train Military populations and to facilitate the increase in frequency of daily practice.

\section{AUTHOR DISCLOSURE STATEMENT}

Marion Trousselard, MD, PhD, is an Active Duty Service Member in the French Army, and was appointed to the NATO panel HFM-195 ("Integrative Medicine Inter- ventions for Military Personnel") as a technical team member for the duration of the Team. Resources from the French Ministry of Defense supported the travel expenses to the team meetings. The author did not receive any funding by governmental or non-governmental organizations to write this report. The author was never employed or paid by any military or religious organization. No competing financial conflicts exist. Frédéric Dutheil, $\mathbf{M D}, \mathbf{P h D}$, is an $\mathrm{MD}-\mathrm{PhD}$ at the University Hospital of Clermont-Ferrand (CHU), France, and a clinical fellow of the Australian Catholic University. His topics are related to biomarkers of stress, metabolism and exercise. He was an associate collaborator in research linked to the NATO panel HFM-195 ("Integrative Medicine Interventions for Military Personnel"). The author did not receive any funding by governmental or non-governmental organizations to write this report. The author was never employed or paid by any military or religious organization. No competing financial conflicts exist. Marie-Hélène Ferrer, $\mathbf{M D}, \mathbf{P h D}$, is an Active Duty Service Member in the French Army. Resources from the French Ministry of Defense supported the travel expenses to the team meetings. The author did not receive any funding by governmental or non-governmental organizations to write this report. The author was never employed or paid by any military or religious organization. No competing financial conflicts exist. Nathalie Babouraj, MD, was at the time of the group meetings an Active Duty Service Member in the French Army, and was appointed to the NATO panel HFM-195 ("Integrative Medicine Interventions for Military Personnel") as a technical team member for the duration of the Team. Resources from the French Ministry of Defense supported the travel expenses to the team meetings. The author did not receive any funding by governmental or non-governmental organizations to write this report. The author was never employed or paid by any military or religious organization. She is now a civilian. No competing financial conflicts exist. Frédéric Canini, MD, PhD, is an Active Duty Service Member in the French Army. Resources from the French Ministry of Defense supported the travel expenses to the team meetings. The author did not receive any funding by governmental or nongovernmental organizations to write this report. The author was never employed or paid by any military or religious organization. No competing financial conflicts exist.

\section{REFERENCES}

1. Morgan CA 3rd, Doran A, Steffian G, Hazlett G, Southwick SM. Stress-induced deficits in working memory and visuoconstructive abilities in special operations soldiers. Biol Psychiatry. 2006;60(7):722-729.

2. Morgan CA 3rd, Wang S, Mason J, et al. Hormone profiles in humans experiencing military survival training. Biol Psychiatry. 2000;47(1):891-901. 
3. Morgan CA 3rd, Wang S, Rasmusson A, Hazlett G, Anderson G, Charney DS. Relationship [sic] among plasma cortisol, catecholamines, neuropeptides $\mathrm{Y}$, and human performance during exposure to uncontrollable stress. Psychosom Med. 2001;63(3):412-422.

4. Ader R. Conditioned immunomodulation: Research needs and directions. Brain Behav Immun. 2003;17(suppl1):S51-S57.

5. Hauger R, Irwin M, Lorang M, Aguilera G, Brown M. High intracerebral levels of $\mathrm{CRH}$ result in $\mathrm{CRH}$ receptor downregulation in the amygdala and neuroimmune desensitization. Brain Res. 1993;676(1-2):283-292.

6. Watkins AD. Perception, emotions and immunity: An integrated homeostatic network. QJM. 1995;88(4):283-294.

7. Roozendahl B, Schoorlemmer G, Koolhaas J, Bohus B. Cardiac, neuroendocrine and behavioral effects of central amygdaloid vassopressinergic and oxytocinergic mechanisms under stress-free conditions in rats. Brain Res Bull. 1993; 32(6):573-579.

8. Porges SW. Cardiac vagal tone: A physiological index of stress. Neurosci Biobehav Rev. 1995;19(2):225-233.

9. Charney DS. Psychobiological mechanisms of resilience and vulnerability: Implications for successful adaptation to extreme stress. Am J Psychiatry. 2004;161(2):195-216.

10. Gray T. Amygdaloid CRF pathways: Role in autonomic, neuroendocrine and behavioral responses to stress. Ann NY Acad. Sci. 1993;697:53-60.

11. Ron de Kloet E, Joëls M, Holsboer F. Stress and the brain: From adaptation to disease. Nat Rev Neurosci. 2005;6(6): 463-475.

12. McCraty R, Atkinson M, Tiller WA, Rein G, Watkins AD. The effects of emotions on short term heart rate variability using power spectrum analysis. Am J Cardiol. 1995; 76(4):1089-1093.

13. McCraty R, Barrios-Choplin B, Rozman D, Watkins D. The impact of a new emotional self-management program on stress, emotions, heart rate variability, DHEA and cortisol. Physiol Behav Sci. 1998;33(2):151-170.

14. Saint-Aubin K. Impact of positive emotions enhancement on physiological processes and psychological functioning in military pilots [presentation]. NATO Research and Technology Organisation Meeting Proceedings MP-HFM-181: Human Performance Enhancement for NATO military operations (Sciences, Technology, and Ethics). Sofia, Bulgaria, Oct 5-7 2009.

15. Friedman BH, Thayer JF. Anxiety and autonomic flexibility: A cardiovascular approach. Biol Psychol. 1998;47(3):243-263.

16. Perraut-Pierre E. Chapter 1. In: Mental Stress Regulation For Performances in Sport [in French]. Paris: Amphora; 2000:5-8.

17. Perraut-Pierre E. Chapter 1. In: Understanding and Practicing the TOP. [in French]. Paris: InterEditions; 2012:23-51.

18. Davis M, Eshelman ER. Chapter 2. In: The Relaxation and Stress Reduction Workbook. Oakland, CA: New Harbinger Publications; 2008:19-27.

19. Seaward BL. Chapter 2. In: Managing Stress: Principles and Strategies for Health and Wellbeing, 5th ed. Burlington: MA: Jones \& Bartlett Learning; 2006:360-368.

20. Crosnier S. Chapter 4. In: Evaluation of the submariners' sleep during a patrol: Interests of the TOP Program $[P h D$ thesis; in French]. Brest, France: University of Medicine of Brest; 2013:90-92.
21. Hoffart MB, Pross KE. The benefits of visualization: Research suggests that visualization promotes relaxation, enhances sleep, reduces pain, and increases creativity. Am J Nurs. 1998;98(12):44-47.

22. Morin CM, Hauri PJ, Espie CA, Spielman AJ, Buysse DJ, Bootzin RR. Non-pharmacologic treatment of chronic insomnia. Sleep. 1999;22(8):1134-1156.

23. Solomon Z, Mikulincer M, Avitzur E. Coping, locus of control, social support, and combat-related post-traumatic stress disorder: A prospective study. J Pers Soc Psychol. 1988;55(2):279-285.

24. Lazarus RS, Folkman S. Chapter 6. In: Stress, Appraisal and Coping. Springer; 1984:141-142.

25. Endler NS, Kantor L, Parker JDA. State-trait coping, statetrait anxiety and academic performance. Pers Indiv Differ. 1994;16(5):663-670.

26. Endler NS, Macrodimitris SD, Kocovski NL. Controllability in cognitive and interpersonal tasks: Is control good for you? Pers Indiv Differ. 2000;29(5):951-962.

27. Penley JA, Tomaka J, Wiebe JS. The association of coping to physical and psychological health outcomes: A meta-analytic review. J Behav Med. 2002;25:551-603.

28. Leifflen D, Arvers P, Lavillunière N. Occupational stress in Paris firefighters: From PTSD to burnout [presentation]. 2nd Franco-American workshop on war traumatism. Val de Grâce School, Paris, March $4^{\text {th }}, 2010$.

29. Cohen S, Karmak T, Mermelstein R. A global measure of perceived stress. J Health Soc Behav. 1983;24(4):385-396.

30. Cerclé A, Gadéa C, Hartmann A, Lourel M. Typological and factor analysis of the perceived stress measure by using the PSS scale. Eur Rev Appl Psychol. 2008;58:227-239.

31. Collange J, Bellinhausen L, Chappé J, Saunder L, Albert E. Perceived stress: When does it become a risk factor for anxiodepressive disorder? [In French]. Arch Mal Prof. 2013; 74:7-15.

32. Shacham S. A shortened version of profile of mood states. $J$ Pers Assess. 1983;47(3):305-306.

33. Trousselard M, Steiler D, Raphel C, Cian C, Duymedjian R, Claverie D, Canini F. Validation of a French version of the Freiburg Mindfulness Inventory-short version: How mindfulness deals with the stress in a working middle-aged population. Biopsychosoc Med. 2010;4(8):1-11.

34. Walach H, Buchheld N, Buttenmüller V, Kleinknecht N, Schmidt S. Measuring mindfulness-the Freiburg Mindfulness Inventory (FMI). Pers Ind Diff. 2006,40:1543-1555.

35. McClelland DC, Alexander C, Marks E. The need for power, stress, immune function, and illness among male prisoners. $J$ Abnorm Psychol. 1982;91(1):61-70.

36. Thompson RA, Asquith P. Quantitation of exocrine $\operatorname{Ig}$ A in human serum in health and disease. Clin Exp Immunol. 1970;7(4):491-500.

37. Schulkin J. Chapter 2. In: Neuroendocrine Regulation of Behavior. Cambridge, UK: Cambridge University Press; 1999:20.

38. Lane EM, Hourani LL, Bray RM, Williams J. Prevalence of perceived stress and mental health indicators among reservecomponent and active-duty military personnel. Am J Public Health. 2012;102(6):1213-1220.

39. Cohen S, Janicki-Deverts D, Miller GE. Psychological stress and disease. JAMA. 2007;298(14):1685-1687. 
40. Trousselard M, Steiler D, Claverie D, Canini F. Relationship between mindfulness and psychological adjustment in soldiers according to their confrontation with repeated deployments and stressors. PSYCH. 2012;3(1):100-115.

41. Vassend O, Watten R, Myhrer T, Syvertsen JL. Negative affectivity and intellectual ability: A study of their relation to self-reported physical symptoms, perceived daily stress and mood, and disciplinary problems in military recruits. Soc Sci Med. 1994;39(4):583-590.

42. Denollet J, De Vries J. Positive and negative affect within the realm of depression, stress and fatigue: The two-factor distress model of the Global Mood Scale (GMS). J Affect Disord. 2006;91(2-3):171-180.

43. Wright CE, Strike PC, Brydon L, Steptoe A. Acute inflammation and negative mood: Mediation by cytokine activation. Brain Behav Immun. 2005;19(4):345-350.

44. Yang Y, Koh D, Ng V, et al. Self-perceived work related stress and the relation with salivary IgA and lysozyme among emergency department nurses. Occup Environ Med. 2002; 59(12):836-841.

45. McCraty R, Atkinson M, Rein G, Watkins AD. Music enhances the effect of positive emotional states on salivary IgA. Stress Med. 1996;12(3):167-175.

46. Lazarus RS, Launier R. Stress-related transactions between person and environment. In: Pervin, LA, Lewis M, eds. Perspectives in Interactional Psychology. New York: Plenum; 1978:287-327.

47. Folkman S, Lazarus RS, Dunkel-Schetter C, DeLongis A, Gruen RJ. Dynamics of a stressful encounter: Cognitive appraisal, coping, and encounter outcomes. J Pers Soc Psychol. 1986;50(5):992-1003.

48. Brown KW, Ryan RM. The benefits of being present: Mindfulness and its role in psychological well-being. J Pers Soc Psychol. 2003;84(4):822-848.
49. Secord PF, Jouard SM. The appraisal of body-cathexis: Bodycathexis and the self. J Consult Clin Psychol. 1953;17(5): 343-347.

50. Bagby RM, Parker, JD, Taylor, GJ. The twenty-item Toronto Alexithymia Scale-I: Item selection and cross validation of factor structure. J Psychosom Res. 1994;38(1):23-32.

51. Bagby RM, Taylor GJ, Parker JD. The twenty-item Toronto Alexithymia Scale-II: Convergent, discriminant, and concurrent validity. J Psychosom Res. 1994;38(1):33-40.

52. Bates M, Bowles S, Hammermeister J, et al. Psychological fitness. Mil Med. 2010;175(8):21-39.

53. Mullen M. On total force fitness in war and peace. Mil Med. 2010;175(8):1.

54. Damasio AR. Chapter 2. In: Descartes' Error: Emotion, Reason, and the Human Brain, New York: Putnam; 1994:112-113.

55. Damasio AR. Chapter 9. In: The Feeling of What Happens: Body and Emotion in the Making of Consciousness. Orlando, FL: Harcourt Brace; 1999:282-285.

56. Damasio AR, Tranel D, Damasio H. Somatic markers and the guidance of behavior: Theory and preliminary testing. In: Levin HS, Eisenberg HM, Bendon AL, eds. Frontal Lobe Function and Dysfunction. Oxford, UK: Oxford University Press; 1991:217-229.

Address correspondence to: Marion Trousselard, MD, PhD Institute of Biomedical Research Armies' Health Service 91223 Brétigny sur Orge Cedex France

E-mail: marion.trousselard@gmail.com 